\title{
An Approach to Estimate the Inherent Deviations by Means of Simultaneous Five-axis Motion
}

\author{
K.M. Muditha Dassanayake ${ }^{1}$, Masaomi TSUTSUMI ${ }^{2}$, Kenji HIGASHIYAMA ${ }^{3}$ and Ken YAMAMOTO \\ 1 Tokyo University of Agriculture and Technology, 2-24-16 Nakacho, Koganei, Tokyo 184-8588, \\ Phone: 042-388-7086, Fax: 042-388-7219 muditha@cc.tuat.ac.jp \\ 2 Tokyo University of Agriculture and Technology \\ ${ }^{3}$ Tokyo University of Agriculture and Technology \\ 4 Tokyo Seimitsu Co. Ltd.
}

\begin{abstract}
This paper presents a methodology for identifying the geometric deviations inherent to tilting rotary table type five-axis machining centers by means of the trajectories of continuous five-axis motions. For identification of the geometric deviations, two sets of reference data are prepared by means of a mathematical model. Four deviations were identified accurately by means of the observation equation in which the ball bar measurements and reference data are applied. The remaining four of the eight deviations was identified accurately by means of simple geometric relations. Furthermore, the effect of the fluctuation and backlash of the worm gear rotation on the accuracy level of the method is discussed. The effectiveness of the proposed methodology as an accuracy test is confirmed by the simulation and the experiment.
\end{abstract}

Keywords: Observation equation, Tilting rotary table type, Geometric relations, Systematic deviation

\section{Introduction}

The tilting rotary table type is used to machine comparatively small and precise work pieces and thus vastly used in industries. This tilting rotary table type is the most common model in Japan.

This paper presents a methodology based on simultaneous five-axis motion which can be used to identify all the eight deviations [1,2] inherent to the tilting rotary table type. Ball bar system is used as a measuring instrument. The methodology consists of two calculation steps which supported by two ball bar measurements, achieved at different height levels from the center of the tilting-axis for the rotary table. To achieve the height levels one extension bar which provided with ball bar system is used. Simple mathematical model is used to carry out the simulations for calculations. The effectiveness of the methodology is confirmed by simulation and experiment.



Fig. 1 Configuration of the machining center
The experimental results show the effectiveness of application to the machining centers. Furthermore, this paper discussed the affect of geometric deviations and the backlash of the worm gear on the accuracy level of the identification procedure and also the affect of work origin on motion trajectory which can be used to compensate the four of eight identified deviations.

\section{Ball bar measurements and simultaneous}

\section{five-axis motion}

\subsection{Ball bar setup and five-axis motion}

In this research work, a simultaneous five-axis control motion is proposed to evaluate all the static deviations inherent to the tilting rotary table type five-axis machining centers, using a ball bar system [3] as a measuring instrument.

The five-axis machine that is the research object is composed of three linear (X, Y and Z) axes and two rotary

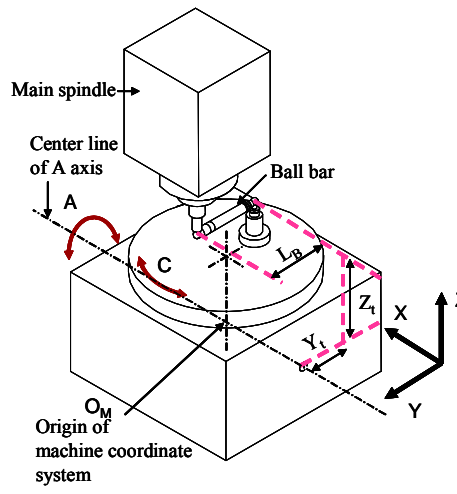

Fig. 2 Setup of ball bar

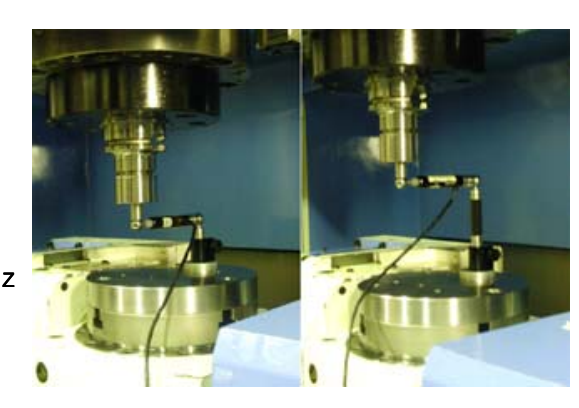

$\begin{array}{ll}\text { (a) at } 150 \mathrm{~mm} & \text { (b) at } 200 \mathrm{~mm}\end{array}$

Fig. 3 Experimental setup 
(A and C) axes as shown in Fig.1. According to the figure, the work table is supported by the C', A' and $\mathrm{Y}^{\prime}$ axes while the spindle head is supported by the $\mathrm{Z}$ and $\mathrm{X}$ axes.

As shown in Fig.2, the ball of the ball bar of the table side is set at $Y_{t}=50 \mathrm{~mm}$ away from the center line of the C' axis in $Y$ direction. The height $Z_{t}$ between the center of the ball of the table side and the A'-axis plays an important role when the static deviations will be identified. The $Y_{t}$ and $Z_{t}$ are the virtual distances measured from the origin of machine coordinate system $\left(\mathrm{O}_{M}\right)$. The other ball is magnetically fixed on the spindle by means of a suitable tool holder as shown in Fig. 2. The distance $L_{B}$ between the two balls is a reference length and shall be calibrated accurately.

In the proposed method, all five axes move at the same time. The $C^{\prime}$-axis rotates from 0 to 360 degrees at constant angular velocity and the $\mathrm{A}^{\prime}$-axis moves from 0 to 90 degrees and then 90 to 0 degrees, while the linear three axes $\mathrm{X}, \mathrm{Y}$ and $\mathrm{Z}$ simultaneously move. During this motion, the distance $L_{B}$ between the two balls of the ball bar is held constant and the sensitive direction of the ball bar is held parallel to the table surface. The point on the center line of the ball bar axis respect to the C' axis is fixed. This motion tests are carried out at two heights of $Z_{t}=150$ and $200 \mathrm{~mm}$. The height was changed by using an extension bar as shown in Fig. 3 .

\subsection{Static deviations inherent to tilting rotary table type}

The deviations inherent to the tilting rotary table type machining centers are determined by the shape generation theory [4]. There are thirteen deviations in the five-axis machine with a tilting rotary table [2]. Among them five deviations are common to the three linear axis machines and those are well measured by the existing techniques provided in ISO 230-1[3]. The remaining eight deviations relate to the rotary axes which are unique features to five-axis machines and thus can be categorized as the inherent deviations of the tilting rotary table type.

The geometric illustration for all the eight deviations is shown in Fig. 4. The four deviations $\left(\delta_{x A Y}, \delta_{y A Y}, \delta_{z A Y}\right.$, and $\alpha_{A Y}$ ) among the eight deviations are considered as adjustable deviations. They can be corrected by adjusting the work coordinates and by adjusting the initial angular position of the $\mathrm{A}^{\prime}$-axis respectively. However, the other deviations $\left(\delta_{y C A}, \beta_{A Y}, \gamma_{A Y}\right.$ and $\left.\beta_{C A}\right)$ can not be corrected.

\subsection{Mathematical model for simulation}

In this research work, we are interesting in the subtraction or elongation of the ball bar during the five-axis motion. Subtraction or elongation of the telescopic ball bar is caused by the static deviations of the linear and rotary axes and other causes such as the fluctuation and backlash of the worm gear. However, the affect of the deviations except for the static deviations is not considered in this model.

In order to make a model for the ball bar, the distance between both centers of the balls of the ball bar are calculated according to the machine coordinate frame (origin $\mathrm{O}_{\mathrm{M}}$ ) by mathematical relations. According to Fig. 2 , the coordinates of the center of the ball of the spindle side can be expressed as in Eq. (1).

$$
\left[\begin{array}{c}
X_{S} \\
Y_{S} \\
Z_{S}
\end{array}\right]=\left[\begin{array}{c}
0 \\
L_{B}-Y_{t} \\
Z_{t}
\end{array}\right]+\left[\begin{array}{c}
X \\
0 \\
Z
\end{array}\right]
$$

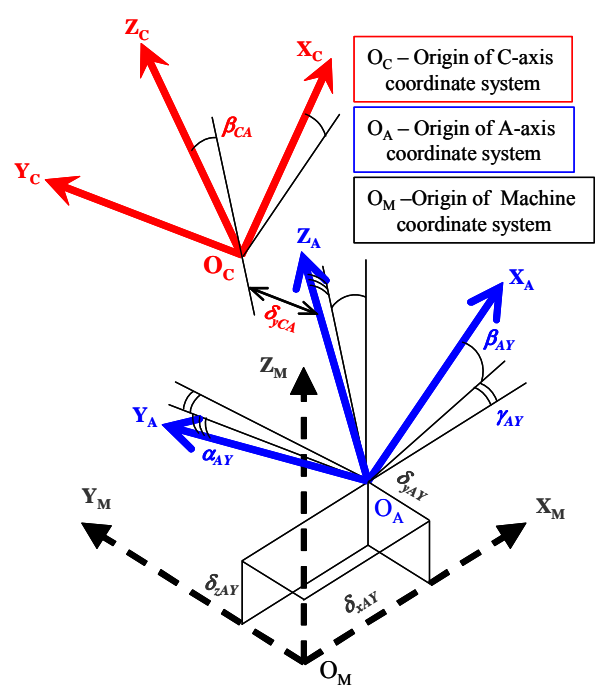

Fig. 4 Geometric illustration of eight deviations

where, $\mathrm{X}$ and $\mathrm{Z}$, which are used in the NC code, are the coordinates of the motion trajectory. By considering Figs. 2 and 4, the static displacement of the center of the ball on the table can be written as,

$\left[\begin{array}{c}\delta_{\text {Xall }} \\ \delta_{\text {Yall }} \\ \delta_{\text {Zall }}\end{array}\right]=\left[\begin{array}{c}0 \\ -Y_{t} \\ Z_{t}\end{array}\right]-\left(D_{\gamma A Y} D_{\beta A Y} D_{\alpha A Y}\left\{D_{\beta C A}\left[\begin{array}{c}0 \\ -Y_{t} \\ Z_{t}\end{array}\right]+\left[\begin{array}{c}0 \\ \delta_{y C A} \\ 0\end{array}\right]\right\}+\left[\begin{array}{c}\delta_{x A Y} \\ \delta_{y A Y} \\ \delta_{z A Y}\end{array}\right]\right)$

where, $D_{\alpha A Y}, D_{\beta A Y}, D_{\gamma A Y}$, and $D_{\beta C A}$ are the matrices for the angular deviations. In general, rotation about $\mathrm{X}, \mathrm{Y}$ and $\mathrm{Z}$ axes can be defined as in Eqs.(3), (4) and (5), respectively.

$$
\begin{aligned}
D_{\alpha} & =\left[\begin{array}{ccc}
1 & 0 & 0 \\
0 & \cos \alpha & -\sin \alpha \\
0 & \sin \alpha & \cos \alpha
\end{array}\right] \\
D_{\beta} & =\left[\begin{array}{ccc}
\cos \beta & 0 & \sin \beta \\
0 & 1 & 0 \\
-\sin \beta & 0 & \cos \beta
\end{array}\right] \\
D_{\gamma} & =\left[\begin{array}{ccc}
\cos \gamma & -\sin \gamma & 0 \\
\sin \gamma & \cos \gamma & 0 \\
0 & 0 & 1
\end{array}\right]
\end{aligned}
$$

Then the coordinates of the center of the ball on the table can be expressed as below.

$\left[\begin{array}{c}X_{T} \\ Y_{T} \\ Z_{T}\end{array}\right]=D_{\gamma A Y} D_{\beta A Y} D_{\alpha A Y} D_{\alpha A}\left\{D_{\beta C A} D_{\gamma C}\left[\begin{array}{c}\delta_{\text {Xall }} \\ -Y_{t}+\delta_{\text {Yall }} \\ Z_{t}+\delta_{\text {zall }}\end{array}\right]+\left[\begin{array}{c}0 \\ \delta_{y C A} \\ 0\end{array}\right]\right\}+\left[\begin{array}{c}\delta_{x A Y} \\ \delta_{y A Y} \\ \delta_{z A Y}\end{array}\right]+\left[\begin{array}{c}0 \\ Y \\ 0\end{array}\right]$ 
where, $D_{\alpha A}$ and $D_{\gamma}$ denote the rotation of the $\mathrm{A}^{\prime}$ and C' axes which are about $\mathrm{X}$ and $\mathrm{Z}$ coordinate axis respectively. $\alpha_{A}, \gamma_{C}$ and $\mathrm{Y}$ are the coordinates of motion trajectory which are given in $\mathrm{NC}$ code.

Thus, the deflection detected by the ball bar can be written as,

$$
e=\sqrt{\left(X_{S}-X_{T}\right)^{2}+\left(Y_{S}-Y_{T}\right)^{2}+\left(Z_{S}-Z_{T}\right)^{2}}-L_{B}
$$

where, $L_{B}$ is the reference length of the ball bar. This mathematical model is used to simulate the effects of static deviations to the motion path. However, to verify the proposed method, simulations have to be carried out with the effect of the deviations other than static deviations. For this reason, the fluctuation, that is a systematic deviation, and the backlash of the worm gear rotation shall be introduced into the mathematical model.

\section{Effect of static deviations on motion trajectories}

\subsection{Effect of static deviations}

The influences of eight geometric deviations on the trajectories are simulated by using the mathematical model expressed by Eq. (7) in order to prepare the reference data for estimating the deviations by the observation equation [1]. For the simulation, the height $Z_{t}$ is $150 \mathrm{~mm}$ and ball bar length $L_{B}$ is $100 \mathrm{~mm}$. Each deviation was applied one by one to the model under the same initial conditions. $0.01 \mathrm{~mm}$ and 0.01 degrees are given to the positional and angular deviations respectively. Figure 5 shows the motion trajectories with each deviation.

The effects of the eight deviations were classified into three groups by observing the shape of the trajectories. One contains the deviations which show unique behaviors as given in Fig. 5(a) and thus each deviation plays a unique role in the trajectories. Therefore, these deviations could be identified by considering the shape of the trajectory. The other two contain the deviations which show two different patterns of trajectories as given in Figs. 5(b) and (c). These two groups show a cumulative effect in the ball bar measurement and thus these deviations may not be identified by considering the shape of the trajectory but the cumulative effect of each group may be identified..

\subsection{Effect of table-ball position}

In general, the angular deviation which is sensitive to the positional relation gives larger displacement than the positional deviation [1]. In order to separate the effect of two geometric deviations which are shown in Figs. 5(b) and (c), simulation was newly conducted by means of the mathematical model in which the height of $Z_{t}$ is set to $200 \mathrm{~mm}$. In this simulation, $0.01 \mathrm{~mm}$ and $0.01^{\circ}$ are also given to the positional and angular deviations, respectively.

An example of the simulation results is shown in Fig. 6. According to the figure, the effect of angular deviations on the five-axis motion path is affected by the distance $Z_{t}$ and its effect increases by $33.3 \%$. This means that the deviation of the five-axis motion path proportionally increases with the increasing height of $Z_{t}$. On the other hand, the positional deviations do not change the deviation of the motion path if the height of $Z_{t}$ changes. Therefore, the relationship between the height of $Z_{t}$ and the cumulative deflections of the two groups, those are given in Figs. 5(b), and (c), could be used to separate the geometric deviations.

\section{Identification method}

\subsection{Identification by simulation data}

Simulations were carried out in the following three cases to clarify the influences of the fluctuation and the backlash of the worm gear rotation on the identification method. Those are,

(a) without fluctuation and backlash of worm gear rotation

(b) with fluctuation of worm gear rotation

(c) with fluctuation and backlash of worm gear rotation

Each simulation was carried out under two inputs at $Z_{t}=150 \mathrm{~mm}$ and $200 \mathrm{~mm}$ by applying all the eight deviations. The values for eight inherent deviations can be selected randomly. For the easy comparison of the identification values, we used the identified values as the input values for first input as given in Table 1. For the second input, the four adjustable deviations which are highlighted were changed as given in Table 1.

\subsubsection{Simulation without fluctuation and backlash of worm gear rotation}

Simulation was carried out by using only the mathematical model in which all the eight deviations were applied. This is carried out for three cases at $Z_{t}=150 \mathrm{~mm}$ and $200 \mathrm{~mm}$. The input values of eight deviations in two cases are given in Table 1. The motion trajectories which are considered as same as the ball bar measurements are illustrated in Fig. 7.

The relationship between each deviation behavior and the cumulative behavior of all deviations which is same as the ball bar measurement is proved as linear by authors [1]. By using this relationship, the solution called Observation equation can be formulated as given below.

$$
\hat{\xi}=\left(V^{T} V\right)^{-1} V^{T} M
$$

The deviations are identified by means of the observation equation. As mentioned above, the observation equation needs two data sets those are the measured data $(M)$ and the reference data $(V)$. The motion trajectories given in Fig. 7 can be considered as the measured data and used in the observation equation accordingly. The reference data can be extracted from the Figs. 5 and 6 . In the cases of $Z_{t}=150 \mathrm{~mm}$ and $200 \mathrm{~mm}$, the reference data should be prepared accordingly.

According to Figs. 5 and 6, there are six patterns in which four are unique. For the unique patterns, we used the same name of deviation which creates that pattern. For the two groups which are given in Figs. 5(b) and (c), we used the names $\delta_{x}$ and $\delta_{y}$, respectively.

The identified data for the cases of $Z_{t}=150 \mathrm{~mm}$ and $200 \mathrm{~mm}$ are given in Table 1. According to the table, 



Fig. 5 Effect of each deviation on motion trajectory

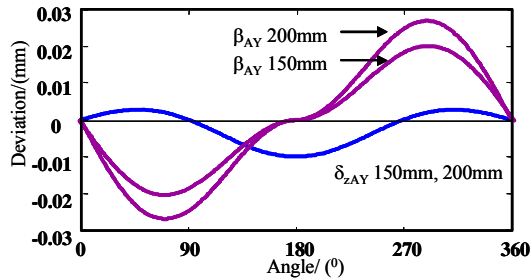

Fig. 6 Represent deviations for the effect of $Z_{t}$ on deviation behaviors

Table 1 Input value sets for simulations

\begin{tabular}{|c|c|c|c|c|c|c|c|}
\hline \multirow[b]{2}{*}{ Deviation } & \multirow{2}{*}{$\begin{array}{c}\text { Deviation } \\
\text { Group }\end{array}$} & \multicolumn{3}{|c|}{ First input } & \multicolumn{3}{|c|}{ Second input } \\
\hline & & input & $\begin{array}{c}Z_{\mathrm{t}} \\
150 \mathrm{~mm}\end{array}$ & $\begin{array}{c}\mathrm{Z}_{\mathrm{t}} \\
200 \mathrm{~mm}\end{array}$ & Input & $\begin{array}{c}Z_{\mathrm{t}} \\
150 \mathrm{~mm}\end{array}$ & $\begin{array}{c}\mathrm{Z}_{\mathrm{t}} \\
200 \mathrm{~mm}\end{array}$ \\
\hline$\delta_{\mathrm{yAY}} /(\mathrm{mm})$ & $\delta_{\mathrm{yAY}} /(\mathrm{mm})$ & 0.0117 & 0.0117 & 0.0117 & -0.0063 & -0.0063 & -0.0063 \\
\hline$\delta_{\mathrm{zAY}} /(\mathrm{mm})$ & $\delta_{\mathrm{zAY}} /(\mathrm{mm})$ & 0.0243 & 0.0243 & 0.0243 & 0.0123 & 0.0123 & 0.0123 \\
\hline$\beta_{\mathrm{AY}} /\left(^{0}\right)$ & $\beta_{\mathrm{AY}} /\left(^{0}\right)$ & 0.0021 & 0.0021 & 0.0021 & 0.0021 & 0.0021 & 0.0021 \\
\hline$\gamma_{\mathrm{AY}} /\left(^{0}\right)$ & $\gamma_{\mathrm{AY}} /\left(^{0}\right)$ & 0.0007 & 0.0007 & 0.0007 & 0.0007 & 0.0007 & 0.0007 \\
\hline$\delta_{\mathrm{xAY}} /(\mathrm{mm})$ & \multirow[t]{2}{*}{$\delta_{\mathrm{x}} /(\mathrm{mm})$} & -0.0030 & \multirow[t]{2}{*}{-0.0018} & \multirow[t]{2}{*}{-0.0014} & -0.0170 & \multirow[t]{2}{*}{-0.0158} & \multirow[t]{2}{*}{-0.0154} \\
\hline$\beta_{\mathrm{CA}} /\left(^{0}\right)$ & & 0.0005 & & & 0.0005 & & \\
\hline$\delta_{\mathrm{yCA}} /(\mathrm{mm})$ & \multirow[t]{2}{*}{$\delta_{\mathrm{y}} /(\mathrm{mm})$} & -0.0031 & \multirow[t]{2}{*}{-0.0084} & \multirow[t]{2}{*}{-0.0102} & -0.0031 & \multirow[t]{2}{*}{0.0178} & \multirow[t]{2}{*}{0.0247} \\
\hline$\alpha_{\mathrm{AY}} /\left(^{0}\right)$ & & 0.0020 & & & -0.0080 & & \\
\hline
\end{tabular}

$\delta_{y A Y}, \delta_{z A Y}, \beta_{A Y}$, and $\gamma_{A Y}$ which show the unique behaviors are identified accurately. The two groups, $\delta_{x}$ and $\delta_{y}$ give different values at $Z_{t}=150 \mathrm{~mm}$ and $200 \mathrm{~mm}$. That is the effect of positional relation on angular deviation. Therefore, we can use the geometric relations to separate the deviations in the two groups.

The geometric relations of the deviations in $\mathrm{YZ}$ plane and ZX plane are illustrated in Fig. 8. According to Fig.8, the relationship between the deviations and $Z_{t}$ can be expressed as below.

$$
\begin{gathered}
\frac{\delta_{y C A}}{\cos \alpha_{A Y}}=Z_{t 1} \tan \alpha_{A Y}+\delta_{y 1} \\
\frac{\delta_{y C A}}{\cos \alpha_{A Y}}=Z_{t 2} \tan \alpha_{A Y}+\delta_{y 2}
\end{gathered}
$$

By manipulating these two equations, relationships for $\alpha_{A Y}$ and $\delta_{y C A}$ can be written as in Eqs. (11) and (12) respectively and thus can be estimated.

$$
\begin{aligned}
& \alpha_{A Y}=\tan ^{-1} \frac{\delta_{y 1}-\delta_{y 2}}{Z_{t 2}-Z_{t 1}} \\
& \delta_{y C A}=\frac{Z_{t 2} \delta_{y 1}-Z_{t 1} \delta_{y 2}}{Z_{t 2}-Z_{t 1}} \cos \alpha_{Y A}
\end{aligned}
$$

The relationship of deviations in $\mathrm{ZX}$ plane and $Z_{t}$ can be formulated as below.

$$
\delta_{x 2}=\delta_{x A Y}+Z_{t 2} \tan \beta_{A C}
$$

From the Eqs. (13) and (14), $\delta_{x A Y}$ and $\beta_{A C}$ can be formulated as in Eqs. (15) and (16) respectively and thus can be estimated.

$$
\begin{gathered}
\delta_{x A Y}=\frac{Z_{t 1} \delta_{x 2}-Z_{t 2} \delta_{x 1}}{Z_{t 1}-Z_{t 2}} \\
\beta_{C A}=\tan ^{-1} \frac{\delta_{x 1}-\delta_{x 2}}{Z_{t 1}-Z_{t 2}}
\end{gathered}
$$

All the identified values of deviations are given in Table 2 . According to the results, all the eight deviations were identified accurately by using two simultaneous five-axis motion trajectory measurements and two sets of simulation data. The adjustable deviations also show the adjustability which can be used to compensate the deviations.

\subsubsection{Simulation with fluctuation of worm gear rotation}

In this section, the influence of fluctuation of worm gear rotation which is categorized as systematic deviation on the identification methodology was examined. To achieve this, the fluctuation of worm gear rotation was introduced into the basic mathematical model by means of the models [5] illustrated in Fig. 9. All the parameters for the models are given in Table 3. $R$ represents the worm gear model which is defined in Eq. (17).

$$
R=R_{\text {all }}+W \sin \left(N R_{\text {all }} \theta\right)
$$

The backlash which is the amount of side to side play in the system is referred to as dead band and is given in Table 3. However in this section backlash is not considered and therefore it is getting as zero. The values of $X_{\text {ref }}, Y_{\text {ref }}, Z_{\text {ref }}, A_{\text {ref }}$ and $C_{r e f}$ are the coordinates which are given in NC code. The $X_{\text {out }}, Y_{\text {out }}, Z_{\text {out }}, A_{\text {out }}$ and $C_{\text {out }}$ are the inputs to the basic mathematical model which are $X, Y, Z$, $\alpha_{A}$ and $\gamma_{C}$ respectively.

The simulation was carried out for three cases at $Z_{t}=150 \mathrm{~mm}$ and $200 \mathrm{~mm}$. The input values for deviations are same as that used in the above. The motion trajectories which are considered as same as the ball bar measurements are given in Fig. 10.

Identification method is same as that used in the above. For the observation equation, the measured data set $(M)$ is extracted from the trajectories given in Fig.10. The reference data set is the same as that used in the above. The identified values of deviations are given in Table 2 .

The results, which given in Table 2 , show that 

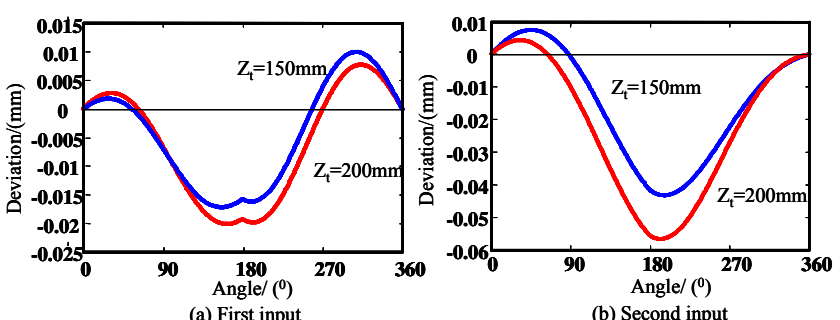

Fig. 7 Motion trajectories without fluctuation and backlash of worm gear

Table 2 All identified deviations by motion trajectories

\begin{tabular}{|c|c|c|c|c|c|c|c|c|}
\hline \multirow[b]{2}{*}{ Deviation } & \multicolumn{4}{|c|}{ First input } & \multicolumn{4}{|c|}{ Second input } \\
\hline & input & Identified & $\begin{array}{l}\text { Identified } \\
\text { (tooth } \\
\text { effect) }\end{array}$ & $\begin{array}{l}\text { Identified } \\
\text { (tooth } \\
\text { effect and } \\
\text { backlash) }\end{array}$ & Input & Identified & $\begin{array}{l}\text { Identified } \\
\text { (tooth } \\
\text { effect) }\end{array}$ & $\begin{array}{l}\text { Identified } \\
\text { (tooth } \\
\text { effect and } \\
\text { backlash) }\end{array}$ \\
\hline$\delta_{\mathrm{YAY}} /(\mathrm{mm})$ & 0.0117 & 0.0117 & 0.0109 & 0.0108 & -0.0063 & -0.0063 & -0.0071 & -0.0072 \\
\hline$\delta_{\mathrm{zAY}} /(\mathrm{mm})$ & 0.0243 & 0.0243 & 0.0251 & 0.0252 & 0.0123 & 0.0123 & 0.0131 & 0.0132 \\
\hline$\beta_{A Y} /\left(^{0}\right)$ & 0.0021 & 0.0021 & 0.0031 & -0.0044 & 0.0021 & 0.0021 & 0.0032 & -0.0043 \\
\hline$\gamma_{A Y} /\left({ }^{0}\right)$ & 0.0007 & 0.0007 & 0.0005 & 0.0070 & 0.0007 & 0.0007 & 0.0005 & 0.0070 \\
\hline$\delta_{\mathrm{XAY}} /(\mathrm{mm})$ & -0.0030 & -0.0030 & -0.0027 & -0.0027 & -0.0170 & -0.0170 & -0.0170 & -0.0170 \\
\hline$\delta_{\mathrm{yCA}} /(\mathrm{mm})$ & -0.0031 & -0.0031 & -0.0030 & -0.0030 & -0.0031 & -0.0031 & -0.0030 & -0.0030 \\
\hline$\alpha_{\mathrm{AY}} /\left(^{\circ}\right)$ & 0.0020 & 0.0020 & 0.0020 & 0.0020 & -0.0080 & -0.0080 & -0.0080 & -0.0080 \\
\hline$\beta_{\mathrm{CA}} /\left(^{0}\right)$ & 0.0005 & 0.0005 & 0.0005 & 0.0005 & 0.0005 & 0.0005 & 0.0004 & 0.0004 \\
\hline
\end{tabular}

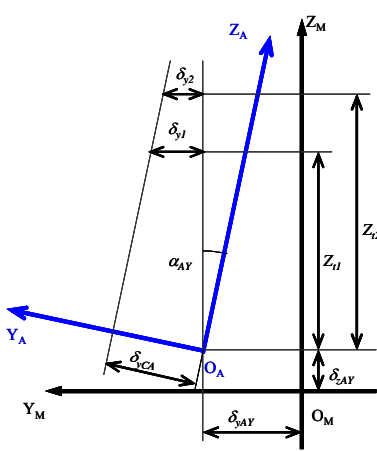

(a) YZ plane

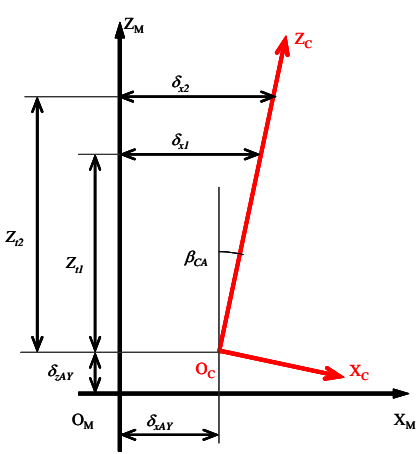

(b) ZX plane
Fig. 8 Geometric relations of deviations

the identification method can be used to identify the deviations within $1 \mu \mathrm{m}$ and $0.001^{\circ}$ accuracy range with the presence of systematic deviations. This is a great achievement because the real trajectory measurements which include the systematic deviations can be used directly to identify the deviations.

\subsubsection{Simulation with fluctuation and backlash of worm gear rotation}

As mentioned above, only the backlash of the $\mathrm{A}^{\prime}$-axis affects to the motion trajectory. Therefore, the backlash of the A'-axis was introduced into the basic mathematical model with the fluctuation of worm gear by means of the models which are given in Fig. 9 as described above. Simulation was carried out for two cases at $Z_{t}=150 \mathrm{~mm}$ and $200 \mathrm{~mm}$. The two inputs to the mathematical model are the same which used in section 4.1.1. The simulated motion trajectories are illustrated in Fig. 11. As illustrated in Fig. 11, A'-axis backlash is appeared at the mid of the cycle of motion trajectory as explained before. And also by comparing the motion trajectories given in Figs.10 and 11, we can say that the
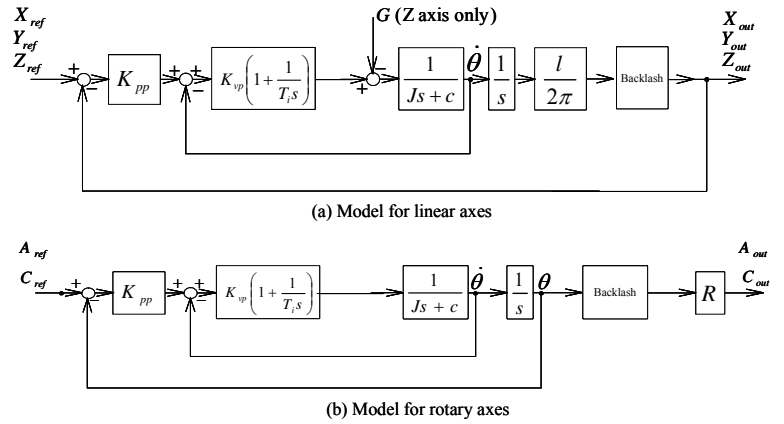

Fig. 9 Models for rotary and linear axes
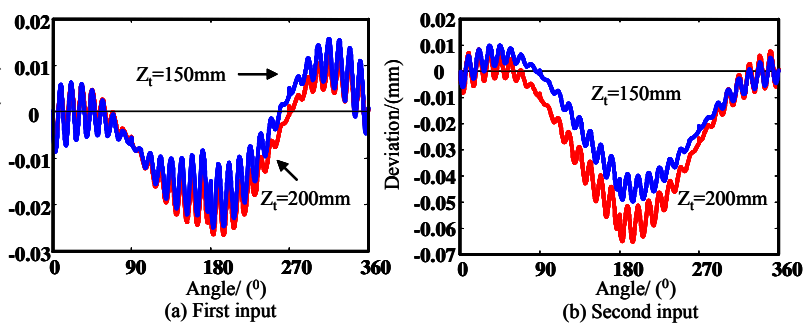

Fig. 10 Motion trajectories with fluctuation of worm gear rotation


Fig. 11 Motion trajectories with fluctuation and backlash of worm gear rotation


Fig. 12 Ball bar measurements of simultaneous five-axis motion backlash of A'-axis affect to the whole motion trajectory.

The eight deviations were identified by applying the same procedure which was used in section 4.1.1. The identified values are given in Table 2. According to the table, six deviations ( $\delta_{X A Y}, \delta_{y A Y}, \delta_{z A Y}, \delta_{y C A}$, $\alpha_{A Y}$, and $\beta_{C A}$ ) can be identified within the range of $1 \mu \mathrm{m}$ and $0.001^{\circ}$ respectively. However the identified values for $\beta_{A Y}$ and $\gamma_{A Y}$ deviate from the input values by $0.007^{\circ}$. That means the backlash of A'-axis affect to the identification method. Therefore we have to adjust the backlash before do the measurements.

\section{Verification by experiments}

The experiment was carried out by using the ball bar as measuring device which was set as illustrated in Fig. 3. Before start taking the measurements, the backlash of A'-axis was compensated by adjusting the machine parameters which provided by manufacture. 
Table 3 Parameters of models for feed drive systems

\begin{tabular}{c|c|c|c|c|c|c}
\hline \multirow{2}{*}{ Parameter } & \multirow{2}{*}{ Unit } & \multicolumn{5}{|c}{ Value } \\
\cline { 3 - 7 } & & X-axis & Y-axis & Z-axis & C-axis & A-axis \\
\hline Equivalent inertia of mechanism, $J$ & $\mathrm{kgm}^{2}$ & 0.008 & 0.0046 & 0.009 & 0.0005 & 0.00005 \\
\hline Viscous damping coefficient, $c$ & $\mathrm{Nms} / \mathrm{rad}$ & 0.04 & 0.035 & 0.03 & 0.0015 & 0.0001 \\
\hline Velocity proportional gain, $K_{v p}$ & $\mathrm{Nms} / \mathrm{rad}$ & 2.8 & 1.61 & 4.5 & 0.05 & 0.05 \\
\hline Velocity integrator reset time, $T_{i}$ & $\mathrm{~s}$ & 0.005 & 0.005 & 0.0057 & 0.1 & 0.1 \\
\hline Position proportional gain, $K_{p p}$ & $\mathrm{rad} / \mathrm{ms}$ & $\begin{array}{c}16500 \\
\text { or } 1 / \mathrm{s}\end{array}$ & $\begin{array}{c}16500 \\
\mathrm{rad} / \mathrm{ms}\end{array}$ & $\begin{array}{c}16500 \\
\mathrm{rad} / \mathrm{ms}\end{array}$ & $\begin{array}{c}\mathrm{rad} / \mathrm{ms} \\
1 / \mathrm{s}\end{array}$ & 42.3 \\
\hline Lead of ball screw, $l$ & $\mathrm{~m}$ & 0.016 & 0.016 & 0.016 & - & - \\
\hline Load torque of gravity, $G$ & $\mathrm{Nm}$ & - & - & 4.19 & - & - \\
\hline Total reduction gear ratio, $R_{a l l}$ & - & - & - & - & $1 / 90$ & $1 / 180$ \\
\hline Number of teeth of worm wheel, $N$ & - & - & - & - & 72 & 60 \\
\hline Fluctuation angle of worm gear, $W$ & $\mathrm{rad}$ & - & - & - & 0.002 & 0.002 \\
\hline Backlash (Dead band width) & - & - & - & - & - & 0.6 \\
\hline
\end{tabular}

Same as simulations, experiment also was conducted for two cases at $Z_{t}=150 \mathrm{~mm}$ and $200 \mathrm{~mm}$. The first two measurements at two height levels were taken immediate after compensate the backlash of A'-axis. The second measurement at two height levels were taken by changing the four adjustable deviations $\left(\delta_{x A Y}, \delta_{y A Y}, \delta_{z A Y}\right.$, and $\alpha_{A Y}$ ) as given in Table 4 . This is done by changing the coordinates of the work origin. The assumed deviation is calculated as below.

(First identification $)+($ Adjusted value $)=($ Assumed deviation $)$

The first identification values and the assumed deviations were used as the input values for all simulations. The ball bar measurements are illustrated in Fig.12. By comparing the simulated motion trajectories which are given in Figs. 7 and 10 with the ball bar measurements in Fig.12, it can be said that the results have a good agreement.

For identifying the deviations, the procedure which used in section 4.1.1 was carried out. The identified values are given in Table 4. According to the table, the positional and angular deviations can be identified within the range of $3 \mu \mathrm{m}$ and $0.001^{\circ}$ respectively. That means this identification method is accurate enough to check the accuracy of the tilting rotary table type five-axis machining centers. And also the results are proved that the coordinates of the origin can be used to compensate the error components which are created by adjustable deviations.

\section{Conclusions}

In this paper, a methodology for identifying the static deviations inherent to tilting rotary table type five-axis machining centers was proposed. This methodology is based on the simultaneous five-axis motion.

Two calculation steps were supported to the method. One is to use of the observation equation and the other is the geometric relations. By using the observation equation, four deviations $\left(\delta_{y A Y}, \delta_{z A Y}, \beta_{A Y}\right.$, and $\left.\gamma_{A Y}\right)$ were identified accurately. The remaining of the eight deviations were identified accurately by using the
Table 4 All identified deviations by experiments

\begin{tabular}{l|c|c|c|c}
\hline \multirow{2}{*}{ Deviation } & \multirow{2}{*}{$\begin{array}{c}\text { First } \\
\text { identification }\end{array}$} & \multicolumn{3}{|c}{ Second identification } \\
\cline { 3 - 5 } & & $\begin{array}{c}\text { Adjusted } \\
\text { value }\end{array}$ & $\begin{array}{c}\text { Assumed } \\
\text { deviation }\end{array}$ & $\begin{array}{c}\text { Identified } \\
\text { value }\end{array}$ \\
\hline$\delta_{\mathrm{yAY}} /(\mathrm{mm})$ & 0.0117 & -0.018 & -0.0063 & -0.0052 \\
\hline$\delta_{\mathrm{zAY}} /(\mathrm{mm})$ & 0.0243 & -0.012 & 0.0123 & 0.0131 \\
\hline$\beta_{\mathrm{AY}} /{ }^{(0)}$ & 0.0021 & - & 0.0021 & 0.0021 \\
\hline$\gamma_{\mathrm{AY}} /\left(^{0}\right)$ & 0.0007 & - & 0.0007 & 0.0004 \\
\hline$\delta_{\mathrm{xAY}} /(\mathrm{mm})$ & -0.0030 & -0.014 & -0.0170 & -0.0140 \\
\hline$\delta_{\mathrm{yCA}} /(\mathrm{mm})$ & -0.0031 & - & -0.0031 & -0.0028 \\
\hline$\left.\alpha_{\mathrm{AY}} /{ }^{0}\right)$ & 0.0020 & -0.01 & -0.0080 & -0.008 \\
\hline$\left.\beta_{\mathrm{CA}} /{ }^{(}\right)$ & 0.0005 & - & 0.0005 & -0.0005 \\
\hline
\end{tabular}

equations which were formulated from the geometric relations. Furthermore, the affect of the fluctuation and backlash of the worm gear on the identification method was discussed. And also the reliability of adjusting the work origin coordinates for compensating four deviations $\left(\delta_{x A Y}, \delta_{y A Y}, \delta_{z A Y}\right.$, and $\left.\alpha_{A Y}\right)$ was proved by simulations and experiment.

The following conclusions were obtained:

(1) The fluctuation of the worm gear rotation does not affect to the identified geometric deviations. However, the backlash of the A'-axis affects to the identified results. Therefore, the measurements have to be taken after the adjustment of backlash.

(2) The four deviations $\left(\delta_{y A Y}, \delta_{z A Y}, \beta_{A Y}\right.$, and $\left.\gamma_{A Y}\right)$ can be identified accurately by means of the observation equation from the measured trajectories.

(3) The remaining of the eight deviations ( $\delta_{x A Y}, \delta_{y A Y}, \delta_{z A Y}$, and $\left.\alpha_{A Y}\right)$ can be identified accurately by means of equations which were formulated from the geometric relations.

\section{References}

[1] Tsutsumi, M.and Saito, A., Identification of angular and positional deviations inherent to 5-axis machining centers with a tilting-rotary table by simultaneous four-axis control movements, International Journal of Machine Tools and Manufacture 44(2004) 1333-1342.

[2] Tsutsumi, M. and Saito, A., Identification and compensation of systematic deviations particular to 5-axis machining centers, International Journal of Machine Tools and Manufacture 43(2003) 771-780.

[3] ISO 230-1: Test code for machine tools - Part 1: Geometric accuracy of machines operating under no-load or finishing conditions, second edition (1996).

[4] Inasaki, I., Kishinami, K., Sakamoto, S., Sugimura, N., Takeuchi, Y., Tanaka, F., Shape generation theory of machine tools-Its basics and applications, Yokendo, Tokyo, (1997), 95-103 (in Japanese).

[5] Sato, R., Yokoburi, Y., Tsutsumi, M., Synchronous accuracy of translational and rotary axes in 5-axis machining centers, Proceedings of The third International Conference on Leading Edge Manufacturing in 21st Century, (Nagoya, Japan), (2005), pp. 993-998. 